PROCEEDINGS OF THE

AMERICAN MATHEMATICAL SOCIETY

Volume 131, Number 11, Pages 3517-3525

S 0002-9939(03)06897-7

Article electronically published on February 20, 2003

\title{
NEHARI AND CARATHÉODORY-FEJÉR TYPE EXTENSION RESULTS FOR OPERATOR-VALUED FUNCTIONS ON GROUPS
}

\author{
MIHÁLY BAKONYI
}

(Communicated by Joseph A. Ball)

\begin{abstract}
Let $G$ be a compact abelian group having the property that its character group $\Gamma$ is totally ordered by a semigroup $P$. We prove that every operator-valued function $k$ on $G$ of the form $k(x)=\sum_{\gamma \in(-P)} \gamma(x) k_{\gamma}$, such that the Hankel operator $H_{k}$ is bounded, has an essentially bounded extension $K$ with $\|K\|_{\infty}=\left\|H_{k}\right\|$. The proof is based on Arveson's Extension Theorem for completely positive functions on $C^{*}$-algebras. Among the corollaries we have a Carathéodory-Fejér type result for analytic operator-valued functions defined on such groups.
\end{abstract}

\section{INTRODUCTION}

In 1911 Carathéodory and Fejér ([7]) proved that given the complex numbers $c_{0}, c_{1}, \cdots, c_{n}$, the minimum of $\|f\|_{\infty}, f$ being a bounded analytic function on the unit disc of the form $f(z)=\sum_{k=0}^{n} c_{k} z^{k}+O\left(z^{n+1}\right)$, equals the norm of the Toeplitz matrix

$$
\left(\begin{array}{cccc}
c_{0} & 0 & \cdots & 0 \\
c_{1} & c_{0} & \cdots & 0 \\
& \ddots & \ddots & \\
c_{n} & c_{n-1} & \cdots & c_{0}
\end{array}\right) .
$$

In 1957 Nehari (12]) proved that, given a sequence of complex numbers $\left\{c_{n}\right\}_{n=-\infty}^{0}$, the minimum over $\|f\|_{\infty}$, when $f$ is an essentially bounded measuarable function on the unit circle $\mathbf{T}$, of the form $f\left(e^{i t}\right)=\sum_{n=-\infty}^{0} c_{n} e^{i n t}+\sum_{n=1}^{\infty} d_{n} e^{i n t}$, equals the norm of the Hankel operator $H_{k}$, where $k(x)=\sum_{n=-\infty}^{0} c_{n} e^{i n t} \cdot\left(H_{k}\right.$ will be defined later in a more general setting.)

The results of Carathéodory and Fejér, respectively Nehari, have been generalized in several directions over the years. These results have been used to solve problems arising from applied sciences (see [9] and the references within). Recently, Nehari's result was generalized in [15] to the case of almost periodic matrix-valued functions which belong to the Wiener class. The authors extended their results for functions of several variables in [16, [17, and [18]. In [8], Nehari's Theorem was

Received by the editors March 6, 2002 and, in revised form, June 16, 2002.

2000 Mathematics Subject Classification. Primary 43A17, 47A57, 43A35, 47A20. 
proved in the case of (scalar) functions defined on a compact abelian group the character group of which is ordered. In the present paper we generalize the latter to the case of operator-valued functions. Since there are no known factorization theorems for operator-valued $H^{1}$ functions on ordered groups, the methods used in the above-mentioned papers cannot be applied for our situation. Our approach is similar to the one used in [4] to prove an operator-valued generalization of Krein's Extension Theorem for ordered groups and makes use of Arveson's Extension Theorem.

The results in this paper are closely related to the recently proved Commutant Lifting Theorem for Ordered Groups [5] (see [6] for particular cases).

For notation and results in group theory and Fourier Analysis on groups we refer to [19] and 20]. We are concerned in this paper with compact abelian groups $G$ on which we consider the normalized Haar measure $m$. For $f \in L^{1}(G)$ (with respect to $m), \hat{f}$ denotes its Fourier transform, defined by

$$
\hat{f}(\gamma)=\int_{G} \overline{\gamma(x)} f(x) d m(x),
$$

for $\gamma \in \Gamma$ (the character group of $G$ ).

Let $\Gamma$ be an abelian group. A function $f: \Gamma \rightarrow \mathcal{L}(\mathcal{H})$ (the algebra of bounded linear operators on a separable Hilbert space $\mathcal{H}$ ) is called positive (semidefinite) if, for every finite subset $\lambda_{1}, \lambda_{2}, \cdots, \lambda_{n} \in \Lambda$, the operator matrix $\left\{f\left(\lambda_{i}-\lambda_{j}\right)\right\}_{i, j=1}^{n}$ is positive (semidefinite).

Suppose $P$ is a semigroup of the abelian group $\Gamma$ and $P$ has the properties

$$
P \cap(-P)=\{e\}, \quad P \cup(-P)=\Gamma .
$$

Under these conditions, $P$ induces a total order on $\Gamma$. If we define $x \geq y$ to mean $x-y \in P$, then the axioms of linear order are satisfied. The choice of a semigroup $P$ with the above properties makes $\Gamma$ into a (totally) ordered group.

In the rest of the paper we consider $G$ to be a compact abelian group the character group $\Gamma$ of which is ordered. It is known that $\Gamma$ can be totally ordered if and only if $G$ is connected. Also, a discrete abelian group can be totally ordered if and only if it is torsion free ([19, Section 8.1).

Let $f \in L^{2}(G) ; f$ is called analytic (antianalytic) if $\hat{f}(\gamma)=0$ for every $\gamma \notin P$ $(\gamma \in P)$. We refer to $f$ as an analytic (antianalytic) polynomial if $f$ is analytic (antianalytic) and $\hat{f}(\gamma)=0$ except for finitely many $\gamma \in P(\gamma \in(-P))$.

Let $L_{G}^{2}(\mathcal{H})$ denote the set of all measurable functions $f: G \rightarrow \mathcal{H}$ such that $\int_{G}\|f(x)\|^{2} d m(x)<\infty$. Then $L_{G}^{2}(\mathcal{H})$ is a Hilbert space under the inner product

$$
(f, g)=\int_{G}(f(x), g(x)) d m(x) .
$$

A function $f \in L_{G}^{2}(\mathcal{H})$ is called analytic (antianalytic) if for every $h \in \mathcal{H}$, the function $f_{h}(x)=(h, f(x))$ is a scalar analytic (antianalytic) function on $G$. The set of all analytic (antianalytic) functions in $L_{G}^{2}(\mathcal{H})$ forms a subspace denoted by $H_{G}^{2}(\mathcal{H})\left(\left[H_{G}^{2}(\mathcal{H})\right]^{\perp}\right)$. If $f \in L_{G}^{2}(\mathcal{H})$, by the linearity of the Fourier transform, for every $\gamma \in \Gamma$ there exists $\hat{f}_{\gamma} \in \mathcal{H}$ such that $\hat{f}_{h}(\gamma)=\left(h, \hat{f}_{\gamma}\right)$, for every $h \in \mathcal{H}$. Then $\|f\|_{2}^{2}=\sum_{\gamma \in \Gamma}\left\|f_{\gamma}\right\|^{2}$ and $f \in H_{G}^{2}(\mathcal{H})$ if and only if $\hat{f}(\gamma)=0$ for every $\gamma \notin P$. 
We denote by $L_{G}^{\infty}(\mathcal{L}(\mathcal{H}))$ the set of all functions $F: G \rightarrow \mathcal{L}(\mathcal{H})$ with the property that for every $h, k \in \mathcal{H},(F(x) h, k)$ is a measurable function and such that $\|F\|_{\infty}<\infty$, where $\|F\|_{\infty}=\operatorname{ess} \sup \{\|F(g)\|, g \in G\}$. A function $F \in L_{G}^{\infty}(\mathcal{L}(\mathcal{H}))$ is called analytic (antianalytic) if for every $h, k \in \mathcal{H}$, the function $(F(x) h, k)$ is a scalar analytic (antianalytic) function on $G$. The set of all such analytic operator-valued functions is denoted by $H_{G}^{\infty}(\mathcal{L}(\mathcal{H}))$. If $F \in L_{G}^{\infty}(\mathcal{L}(\mathcal{H}))$, the operator $M_{F}: L_{G}^{2}(\mathcal{H}) \rightarrow L_{G}^{2}(\mathcal{H})$, defined by $\left(M_{F} g\right)(x)=F(x) g(x)$, for $g \in L_{G}^{2}(\mathcal{H})$, is called the multiplication operator with symbol $F$. It is known that $\left\|M_{F}\right\|=\|F\|_{\infty}$.

By $L_{G}^{2}(\mathcal{L}(\mathcal{H}))$ we denote the set of all functions $F: G \rightarrow \mathcal{L}(\mathcal{H})$ such that for every $h, k \in \mathcal{H},(F(x) h, k) \in L^{2}(G)$. If $F \in L_{G}^{2}(\mathcal{L}(\mathcal{H}))$, then for every $\gamma \in \Gamma$ there exists $F_{\gamma} \in \mathcal{L}(\mathcal{H})$ such that $F(x)=\sum_{\gamma \in \Gamma} \gamma(x) F_{\gamma}$ (the convergence is in the weak sense). The latter is known for the matrix-valued case 10, but a construction similar to the one for $L_{G}^{2}(\mathcal{H})$ in a previous paragraph proves it for the operator-valued case also. Let $k \in L_{G}^{2}(\mathcal{L}(\mathcal{H}))$ be of the form $k(x)=\sum_{\gamma \in(-P)} \gamma(x) k_{\gamma}$. Define the (possibly unbounded) operator $H_{k}: H_{G}^{2}(\mathcal{H}) \rightarrow\left[H_{G}^{2}(\mathcal{H})\right]^{\perp}$ by $H_{k} f=P_{-} M_{k} f$, where $P_{-}$is the orthogonal projection of $L_{G}^{2}(\mathcal{H})$ onto $\left[H_{G}^{2}(\mathcal{H})\right]^{\perp}$. The operator $H_{k}$ is referred to as the Hankel operator with symbol $k$.

For $K \in L_{G}^{1}(\mathcal{L}(\mathcal{H}))$ and $h, k \in \mathcal{H}$, define $K_{h, k}(x)=(K(x) h, k)$. By the linearity of the Fourier transform, for every $\gamma \in \Gamma$ there exists $\hat{K}(\gamma) \in \mathcal{L}(\mathcal{H})$, such that $(\hat{K}(\gamma) h, k)=\hat{K}_{h, k}(\gamma)$, for $h, k \in \mathcal{H}$. It is known that $\hat{K}(\gamma) \neq 0$ for only at most countably many $\gamma \in \Gamma$.

The following is the main result of the paper.

Theorem 1.1. Let $G$ be a compact abelian group the character group $\Gamma$ of which has an order induced by a semigroup $P$. Let $k \in L_{G}^{2}(\mathcal{L}(\mathcal{H}))$ of the form $k(x)=$ $\sum_{\gamma \in(-P)} \gamma(x) k_{\gamma}$ be such that $H_{k}$ is bounded. Then there exists $K \in L_{G}^{\infty}(\mathcal{L}(\mathcal{H}))$, such that $\|K\|_{\infty}=\left\|H_{k}\right\|$ and $\hat{K}(\gamma)=k_{\gamma}$, for every $\gamma \in(-P)$.

Before proving Theorem 1.1 we need some lemmas. The first lemma is the necessary part of the operator-valued version of Bochner's Theorem.

Lemma 1.2. Let $F \in L_{G}^{1}(\mathcal{L}(\mathcal{H})), F(g) \geq 0$ for $g \in G$ a.e. Then $\hat{F}: \Gamma \rightarrow \mathcal{L}(\mathcal{H})$ is positive definite.

Proof. Let $\left\{\gamma_{i}\right\}_{i=1}^{n} \subset \Gamma$ and $\left\{h_{i}\right\}_{i=1}^{n} \subset \mathcal{H}$. Then

$$
\begin{gathered}
\sum_{i, j=1}^{n}\left(\hat{F}\left(\gamma_{i}-\gamma_{j}\right) h_{i}, h_{j}\right)=\sum_{i, j=1}^{n} \int_{G} \gamma_{i}(g) \overline{\gamma_{j}(g)}\left(F(g) h_{i}, h_{j}\right) d m(g) \\
=\int_{G}\left(F(g) \sum_{i=1}^{n} \gamma_{i}(g) h_{i}, \sum_{i=1}^{n} \gamma_{i}(g) h_{i}\right) d m(g) \geq 0 .
\end{gathered}
$$

This implies that the operator matrix $\left\{\hat{F}\left(\gamma_{i}-\gamma_{j}\right)\right\}_{i, j=1}^{n}$ is positive definite, thus $\hat{F}$ is a positive definite function.

Lemma 1.3. Let $\Phi: \Gamma \rightarrow \mathcal{L}(\mathcal{H})$ be a positive definite function with finite support on the character group $\Gamma$ of a compact abelian group $G$. Then $\sum_{\gamma \in \Gamma} \Phi(\gamma) \geq 0$. 
Proof. Let $h \in \mathcal{H}$ and define $\Phi_{h}(\gamma)=(\Phi(\gamma) h, h)$. Then $\Phi$ is a positive definite scalar function. By Theorem 1.9.8 in [20],

$$
\check{\Phi}_{h}(e)=\sum_{\gamma \in \Gamma} \Phi_{h}(\gamma)=\left(\sum_{\gamma \in \Gamma} \Phi(\gamma) h, h\right) \geq 0 .
$$

Since (1) holds for every $h \in \mathcal{H}$, we have that $\sum_{\gamma \in \Gamma} \Phi(\gamma) \geq 0$.

Lemma 1.4. Let $k \in L_{G}^{2}(\mathcal{L}(\mathcal{H})), k(x)=\sum_{\gamma \in(-P)} \gamma(x) k_{\gamma}$ be such that $\left\|H_{k}\right\| \leq 1$.

Then for every $\left\{\gamma_{i}\right\}_{i=1}^{n} \subset \Gamma, \gamma_{1} \leq \gamma_{2} \leq \cdots \leq \gamma_{n}$, the operator matrix

$$
\left(\begin{array}{cccc}
k_{\gamma_{1}-\gamma_{i}} & k_{\gamma_{2}-\gamma_{i}} & \cdots & k_{e} \\
k_{\gamma_{1}-\gamma_{i+1}} & k_{\gamma_{2}-\gamma_{i+1}} & \cdots & k_{\gamma_{i}-\gamma_{i+1}} \\
\vdots & \vdots & \vdots & \vdots \\
k_{\gamma_{1}-\gamma_{n}} & k_{\gamma_{2}-\gamma_{n}} & \cdots & k_{\gamma_{i}-\gamma_{n}}
\end{array}\right)
$$

is a contraction for every $i=1, \cdots, n$. (For $i=1$ the matrix reduces to a column while for $i=n$ it reduces to a row.)

Proof. The matrix (2) represents the compression of $H_{k}$ to the subspaces $\{f \in$ $H_{G}^{2}(\mathcal{H}): \hat{f}(\gamma)=0$ for $\left.\gamma \notin\left\{\gamma_{i}-\gamma_{1}, \cdots, \gamma_{i}-\gamma_{i-1}, 0\right\}\right\}$ and $\left\{f \in\left[H_{G}^{2}(\mathcal{H})\right]^{\perp}: \hat{f}(\gamma)=0\right.$, for $\left.\gamma \notin\left\{0, \gamma_{i}-\gamma_{i+1}, \cdots, \gamma_{i}-\gamma_{n}\right\}\right\}$.

Remark. It can be shown that $\left\|H_{k}\right\| \leq 1$ if and only if all matrices (2) are contractive for every $\left\{\gamma_{i}\right\}_{i=1}^{n} \subset \Gamma$. Similarly to the definition of a positive definite function, one might call a function $k:(-P) \rightarrow \mathcal{L}(\mathcal{H}), k(\gamma)=k_{\gamma}$, such that $\left\|H_{k}\right\| \leq 1$, as contractive definite on $(-P)$.

The following result might be known, but for sake of completeness, we present it with a proof.

Proposition 1.5. Let $G$ be a compact group and let $L: C(G) \rightarrow \mathcal{L}(\mathcal{H})$ be such that $\|L(p)\| \leq\|p\|_{1}$, for every $p \in C(G)$. Then there exists a function $Q \in L_{G}^{\infty}(\mathcal{L}(\mathcal{H}))$, such that $\|Q\|_{\infty} \leq 1$, and

$$
(L(p) h, k)=\int_{G} p(x)(Q(x) h, k) d m(x)
$$

for every $p \in C(G)$ and $h, k \in \mathcal{H}$.

Proof. For fixed $h, k \in \mathcal{H}, \alpha_{h, k}(p)=(L(p) h, k)$ is a functional on $C(G)$ such that $\left|\alpha_{h, k}(p)\right| \leq\|h\|\|k\|\|p\|_{1}$. By the Hahn-Banach Theorem, $\alpha$ can be extended to a bounded linear functional on $L^{1}(G)$ of norm $\leq\|h\|\|k\|$. Thus there exists $f_{h, k} \in$ $L^{\infty}(G),\left\|f_{h, k}\right\|_{\infty} \leq\|h\|\|k\|$ such that

$$
(L(p) h, k)=\int_{G} p(x) f_{h, k}(x) d m(x),
$$

for every $p \in C(G)$. Since $\mathcal{H}$ is separable, (4) implies that for almost every $x \in G$, the mapping $(h, k) \in \mathcal{H} \times \mathcal{H} \rightarrow f_{h, k}(x)$ is linear in $h$, antilinear in $k$, and $\left|f_{h, k}(x)\right| \leq$ $\|h\|\|k\|$. Thus for such $x$ there exists $Q(x) \in \mathcal{L}(\mathcal{H})$ such that $f_{h, k}(x)=(Q(x) h, k)$. We can extend this function to a function defined on $G,\|Q\|_{\infty} \leq 1$, and then (4) implies (3) and the proof is complete. 
Remark. It is known that $\mathcal{L}(\mathcal{H})$ does not have the Radon-Nikodym property. This fact does not conflict with Proposition 1.5, since our notion of measurability is weaker than the ones usually considered in connection with vector measures.

If $\mathcal{M}_{n}$ denotes the algebra of all $n \times n$ complex matrices, the tensor product $\mathcal{M}_{n} \otimes \mathcal{M}_{m}$ can alternatively be identified either with $n \times n$ matrices with entries in $\mathcal{M}_{m}$, or with $m \times m$ matrices with entries in $\mathcal{M}_{n}$. The isomorphism between these identifications is called the canonical shuffle ([13]). Actually, it will be used below in a more general sense, namely when the entries are operators rather than scalars.

\section{Proof of Theorem 1.1}

Proof. If $H_{k}=0$, then we can choose $K=0$ and the result follows immediately. If $\left\|H_{k}\right\|>0$, we can, without loss of generality, assume that $\left\|H_{k}\right\|=1$.

Let $\mathcal{S}$ be the set of all $2 \times 2$ matrix-valued functions of the form $\left(\frac{p}{q} q\right)$, where $p \in C(G)$ and $q$ is an antianalytic polynomial on $G$. For $\left(\frac{p}{q} \frac{q}{p}\right) \in \mathcal{S}$, define $\Phi$ :

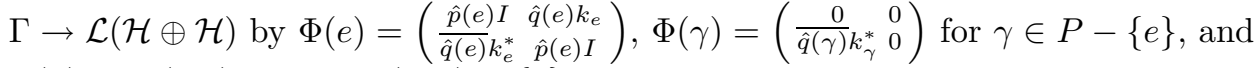
$\Phi(\gamma)=\Phi(-\gamma)^{*}$ for $\gamma \in(-P)-\{e\}$. We first prove that $\Phi$ is a positive definite function on $\Gamma$ whenever $\left(\frac{p(x)}{q(x)} p(x)=\geq 0\right.$ for all $x \in G$. We have to show that for any $\left\{\gamma_{i}\right\}_{i=1}^{n} \subset \Gamma, \gamma_{1} \leq \gamma_{2} \leq \cdots \leq \gamma_{n}$, the operator matrix $\left\{\Phi\left(\gamma_{i}-\gamma_{j}\right)\right\}_{i, j=1}^{n}$ is positive (semidefinite). By canonically reshuffling the matrix $\left\{\Phi\left(\gamma_{i}-\gamma_{j}\right)\right\}_{i, j=1}^{n}$, one obtains the operator matrix $\left(\begin{array}{cc}\hat{p}(e) I_{n} & C \\ C^{*} & \hat{p}(e) I_{n}\end{array}\right)$, where $I_{n}$ is the identity operator on $\bigoplus_{i=1}^{n} \mathcal{H}$ and

$$
C=\left(\begin{array}{cccc}
\hat{q}(e) k_{e} & 0 & \cdots & 0 \\
\hat{q}\left(\gamma_{1}-\gamma_{2}\right) k_{\gamma_{1}-\gamma_{2}} & \hat{q}(e) k_{e} & \cdots & 0 \\
\vdots & \vdots & \vdots & \vdots \\
\hat{q}\left(\gamma_{1}-\gamma_{n}\right) k_{\gamma_{1}-\gamma_{n}} & \hat{q}\left(\gamma_{2}-\gamma_{n}\right) k_{\gamma_{2}-\gamma_{n}} & \cdots & \hat{q}(e) k_{e}
\end{array}\right) .
$$

Let $A=\left\{A_{i j}\right\}_{i, j=1}^{n}$ be the lower triangular partial operator matrix defined by

$$
\left(A_{i j}\right)_{i, j=1}^{n}=\left\{\begin{array}{l}
k\left(\gamma_{i}-\gamma_{j}\right) \text { for } i \geq j, \\
\text { unspecified for } i<j .
\end{array}\right.
$$

By Lemma 1.4 all fully specified submatrices of $A$ are contractions. By Arveson's Distance Formula [3], $A$ admits a contractive extension $B$. Then $\left(\begin{array}{c}I \\ B^{*}\end{array}\right.$

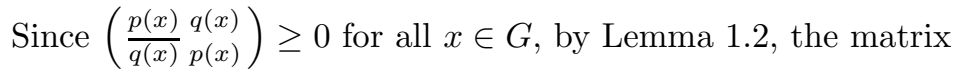

$$
Z=\left(\begin{array}{cc}
\left\{\hat{p}\left(\gamma_{i}-\gamma_{j}\right)\right\}_{i, j=1}^{n} & \left\{\hat{q}\left(\gamma_{i}-\gamma_{j}\right)\right\}_{i, j=1}^{n} \\
\left(\left\{\hat{q}\left(\gamma_{i}-\gamma_{j}\right)\right\}_{i, j=1}^{n}\right)^{*} & \left\{\hat{p}\left(\gamma_{i}-\gamma_{j}\right\}_{i, j=1}^{n}\right.
\end{array}\right)
$$

is positive. The matrix $\left(\begin{array}{cc}\hat{p}(e) I_{n} & C \\ C^{*} & \hat{p}(e) I_{n}\end{array}\right)$ is the Schur product of $\left(\begin{array}{cc}I & B \\ B^{*} & I\end{array}\right)$ and $Z$ (the unspecified positions in $A$ correspond to zeros in $Z$ ). By a generalized version of Schur's Theorem (14, proof of Theorem 4.3), it follows that $\left(\begin{array}{cc}\hat{p}(e) I_{n} & C \\ C^{*} & \hat{p}(e) I_{n}\end{array}\right) \geq 0$, implying that $\Phi$ is a positive function on $\Gamma$. 
By Lemma 1.3 the function $l: \mathcal{S} \rightarrow \mathcal{L}(\mathcal{H} \oplus \mathcal{H})$, defined by

$$
l\left(\left(\begin{array}{cc}
p & q \\
\bar{q} & p
\end{array}\right)\right)=\sum_{\gamma \in \Gamma} \Phi(\gamma)=\left(\begin{array}{cc}
\hat{p}(e) I & \sum_{\gamma \in \Gamma} \hat{q}(\gamma) k_{\gamma} \\
\sum_{\gamma \in \Gamma} \overline{\hat{q}(\gamma)} k_{\gamma}^{*} & \hat{p}(e) I
\end{array}\right),
$$

is positive.

We will prove that $l$ is completely positive. For $n \geq 1$ denote $\mathcal{S}_{n}=$ $\mathcal{S} \otimes \mathcal{M}_{n}$. For $\left\{\left(\frac{p_{i j} q_{i j}}{q_{i j} p_{i j}}\right)\right\}_{i, j=1}^{n} \in \mathcal{S}_{n}$, define the functions $U_{i j}: \Gamma \rightarrow \mathcal{L}(\mathcal{H} \oplus \mathcal{H})$,

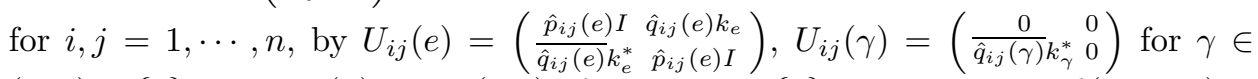
$(-P)-\{e\}$, and $U_{i j}(\gamma)=U_{i j}(-\gamma)^{*}$ for $\gamma \in P-\{e\}$. Let $U: \Gamma \rightarrow \mathcal{L}(\mathcal{H} \oplus \mathcal{H}) \otimes$ $\mathcal{M}_{n}, U(\gamma)=\left\{U_{i j}(\gamma)\right\}_{i, j=1}^{n}$. We prove that $U$ is positive definite on $\Gamma$ whenever $\left\{\left(\frac{p_{i j}(x) q_{i j}(x)}{\bar{q}_{i j}(x)} p_{i j}(x)\right)\right\}_{i, j=1}^{n} \geq 0$ for all $x \in G$. We have to show that for any $\left\{\gamma_{k}\right\}_{k=1}^{m} \subset \Gamma$, $\gamma_{1} \leq \gamma_{2} \leq \cdots \leq \gamma_{m}$, the operator matrix $\left\{U\left(\gamma_{l}-\gamma_{k}\right)\right\}_{k, l=1}^{m}$ is positive. Applying the canonical shuffle to $\left\{U\left(\gamma_{l}-\gamma_{k}\right)\right\}_{k, l=1}^{m}$, one obtains the operator matrix

$$
\left\{\left(\begin{array}{cc}
\hat{p}_{i j}(e) I_{m} & C_{i j} \\
C_{i j}^{*} & \hat{p}_{i j}(e) I_{m}
\end{array}\right)\right\}_{i, j=1}^{n} \odot\left(\left(\begin{array}{cc}
I & B \\
B^{*} & I
\end{array}\right) \otimes J_{n}\right),
$$

where $I_{m}$ is the identity matrix on $\bigoplus_{i=1}^{m} \mathcal{H}$,

$$
C_{i j}=\left(\begin{array}{cccc}
\hat{q}_{i j}(e) k_{e} & 0 & \cdots & 0 \\
\hat{q}_{i j}\left(\gamma_{1}-\gamma_{2}\right) k_{\gamma_{1}-\gamma_{2}} & \hat{q}_{i j}(e) k_{e} & \cdots & 0 \\
\vdots & \vdots & \vdots & \vdots \\
\hat{q}_{i j}\left(\gamma_{1}-\gamma_{n}\right) k_{\gamma_{1}-\gamma_{n}} & \hat{q}_{i j}\left(\gamma_{2}-\gamma_{n}\right) k_{\gamma_{2}-\gamma_{n}} & \cdots & \hat{q}_{i j}(e) k_{e}
\end{array}\right),
$$

$\odot$ denotes the Schur product, $J_{n}$ is the $n \times n$ matrix with all entries equal to 1 , and $B$ is the contractive extension of the partial matrix $A$ defined by (5). Then (6) and the generalized version of Schur's Theorem (14]) imply that $\left\{U\left(\gamma_{k}-\gamma_{l}\right)\right\}_{k, l=1}^{m}$ is positive semidefinite, thus $U$ is a positive definite function. By Lemma 1.3, the function $l_{n}: \mathcal{S}_{n} \rightarrow \mathcal{L}(\mathcal{H} \oplus \mathcal{H}) \otimes \mathcal{M}_{n}$, defined by

$$
l_{n}\left(\left\{\left(\begin{array}{cc}
p_{i j} & q_{i j} \\
\bar{q}_{i j} & p_{i j}
\end{array}\right)\right\}_{i, j=1}^{n}\right)=\sum_{\gamma \in \Gamma} U(\gamma)=\left\{\left(\begin{array}{cc}
\hat{p}_{i j}(e) I & \sum_{\gamma \in \Gamma} \hat{q}_{i j}(\gamma) k_{\gamma} \\
\sum_{\gamma \in \Gamma} \overline{\hat{q}_{i j}(\gamma)} k_{\gamma}^{*} & \hat{p}_{i j}(e) I
\end{array}\right)\right\}_{i, j=1}^{n},
$$

is positive, implying that $l$ is a completely positive function on $\mathcal{S}$. Since $\mathcal{S}$ is an operator system in $C(G) \otimes \mathcal{M}_{2}$, by Arveson's Theorem ([2], see also Theorem 6.5 in [13]), $l$ admits a (completely) positive extension $L: C(G) \otimes \mathcal{M}_{2} \rightarrow \mathcal{L}(\mathcal{H} \oplus \mathcal{H})$.

Let $p \in C(G)$. Since $|\hat{p}|(e)=\int_{G}|p(x)| d m(x)=\|p\|_{1}$,

$$
L\left(\left(\begin{array}{cc}
|p| & 0 \\
0 & |p|
\end{array}\right)\right)=\left(\begin{array}{cc}
\|p\|_{1} I & 0 \\
0 & \|p\|_{1} I
\end{array}\right) .
$$

Let $L\left(\left(\begin{array}{ll}0 & p \\ 0 & 0\end{array}\right)\right)=\left(\begin{array}{ll}L_{11}(p) & L_{12}(p) \\ L_{21}(p) & L_{22}(p)\end{array}\right)$. Since $\left(\begin{array}{cc}|p(x)| & \pm p(x) \\ \pm p(x) & |p(x)|\end{array}\right) \geq 0$ for $x \in G$, we have that

$$
\left(\begin{array}{cc}
\|p\|_{1} I & 0 \\
0 & \|p\|_{1} I
\end{array}\right) \pm\left(\begin{array}{cc}
L_{11}(p)+L_{11}(p)^{*} & L_{12}(p)+L_{21}(p)^{*} \\
L_{12}(p)^{*}+L_{21}(p) & L_{22}(p)+L_{22}(p)^{*}
\end{array}\right) \geq 0,
$$

implying that

$$
\left\|L_{12}(p)+L_{21}(p)^{*}\right\| \leq\|p\|_{1}
$$


Since $\left(\begin{array}{cc}|p(x)| & \pm i p(x) \\ \mp i p(x) & |p(x)|\end{array}\right) \geq 0$ for $x \in G$, we have that

$$
\left(\begin{array}{cc}
\|p\|_{1} I & 0 \\
0 & \|p\|_{1} I
\end{array}\right) \pm\left(\begin{array}{cc}
i\left(L_{11}(p)-L_{11}(p)^{*}\right) & i\left(L_{12}(p)-L_{21}(p)^{*}\right) \\
i\left(L_{12}(p)^{*}-L_{21}(p)\right) & i\left(L_{22}(p)-L_{22}(p)^{*}\right)
\end{array}\right) \geq 0
$$

implying that

$$
\left\|L_{12}(p)-L_{21}(p)^{*}\right\| \leq\|p\|_{1} .
$$

By (77) and (8) we get that $\left\|L_{12}(p)\right\| \leq\|p\|_{1}$.

By Proposition 1.5 there exists $Q \in L_{G}^{\infty}(\mathcal{L}(\mathcal{H}))$ such that $\|Q\|_{\infty} \leq 1$ and

$$
\left(L_{12}(p) h, k\right)=\int_{G} p(x)(Q(x) h, k) d m(x),
$$

for every $h, k \in \mathcal{H}$. Let $\gamma_{0} \in(-P)$ and define $p_{0}(x)=\gamma_{0}(x)$. Since $L_{12}$ is an extension of the mapping that assigns to every antianalytic polynomial $p$ the operator $\sum_{\gamma \in \Gamma} \hat{p}(\gamma) k_{\gamma}$, we have that

$$
\left(k_{\gamma_{0}} h, k\right)=\left(L_{12}\left(p_{0}\right) h, k\right)=\int_{G} \gamma_{0}(x)(Q(x) h, k) d m(x) .
$$

Define $K(x)=Q(-x)$. Then (9) implies that $k_{\gamma}=\hat{K}(\gamma)$, for every $\gamma \in(-P)$. Since $H_{K}=H_{k}$, we have that $\|K\|_{\infty}=\left\|M_{K}\right\| \geq\left\|H_{K}\right\|=1$, and this completes the proof.

\section{Corollaries of Theorem 1.1}

We first mention some particular cases of ordered groups that have been considered earlier.

Let $G$ be a locally compact abelian group and let $\Gamma$ be its character group. Let $\Gamma_{d}$ be the group $\Gamma$ with the discrete topology, and $\bar{G}$ be the character group of $\Gamma_{d}$. Then $\bar{G}$ is a compact abelian group called the Bohr compactification of $G$ ([19], 1.8.1). Theorem 1.1 can be applied to any such $\bar{G}$.

When $\Gamma=\mathbf{R}_{d}$, then $G=\overline{\mathbf{R}}$ and the continuous functions on $\overline{\mathbf{R}}$ are referred to in the literature as almost periodic functions. Unfortunately, when $k$ is an almost periodic function, there might not exist any almost periodic function $K$ such that $\|K\|_{\infty}=\left\|H_{k}\right\|$. (As shown by a counterexample in [1, the latter fails even for scalar continuous functions on the unit circle.) This is why in [15]-[18], the (matrix-valued) function $k$ was considered to belong to the Wiener class (i.e., $\left.\sum_{\gamma<0}\left\|k_{\gamma}\right\|<\infty\right)$. It was proven in that case that, if $\left\|H_{k}\right\|<1$, there exists an almost periodic extension $K$ of $k, K$ in the Wiener class, such that $\|K\|_{\infty}<1$. The authors also succeeded in parametrizing in the matrix-valued case the set of all such extensions $K$.

However, Theorem 1.1 implies that if $k: \overline{\mathbf{R}} \rightarrow \mathcal{L}(\mathcal{H})$ is of the form $k(x)=$ $\sum_{n=-\infty}^{0} e^{i \gamma_{n} x} k_{n}, \gamma_{n} \leq 0$ for every $n \leq 0$, and $\left\|H_{k}\right\|<\infty$, then $k$ has an extension $K(x)=\sum_{n=-\infty}^{\infty} e^{i \gamma_{n} x} k_{n}, \gamma_{n}>0$ for $n>0$, with $\|K\|_{\infty}=\left\|H_{k}\right\|$. The convergence of the latter series is in the $L^{2}$ sense which is weaker than the $l^{1}$ convergence in the Wiener algebra case. 
If $G=\mathbf{T}^{r}$ (the $r$-dimensional torus), then $\Gamma=\mathbf{Z}^{r}$. Let $\alpha_{1}, \alpha_{2}, \cdots, \alpha_{r} \geq 0$ be given. Consider in $\mathbf{Z}^{r}$ the set

$$
P=\left\{\left(m_{1}, \cdots, m_{r}\right): \alpha_{1} m_{1}+\cdots+\alpha_{r} m_{r}>0\right\}
$$

$$
\cup\left\{\left(m_{1}, \cdots, m_{r}\right): \alpha_{1} m_{1}+\cdots+\alpha_{r} m_{r}=0, m_{1}=m_{2}=\cdots=m_{k}=0, m_{k+1}>0\right\} .
$$

Then $P$ defines an order on $\mathbf{Z}^{r} ; P$ is referred to as a Helson-Lowdenslager half-space [10]. A particular case of such $P$ is the lexicographic order on $\mathbf{Z}^{r}$.

A result similar to Theorem 1.1 for the case of matrix-valued functions in the Wiener class and $P$ as in (10) was proved in [16], together with a parametrization of the set of all strictly contractive solutions.

The scalar versions of the next two corollaries were proved in [8], while in 16] a proof based on the Commutant Lifting approach can be found for the matrix-valued version of Corollary 3.1

Corollary 3.1. For every $k \in L_{G}^{\infty}(\mathcal{L}(\mathcal{H}))$, $\operatorname{dist}\left(k, H_{G}^{\infty}(\mathcal{L}(\mathcal{H}))\right)=\left\|H_{k}\right\|$. Moreover, the distance is attained.

Proof. If $k \in L_{G}^{\infty}(\mathcal{L}(\mathcal{H}))$ and $f \in H_{G}^{\infty}(\mathcal{L}(\mathcal{H}))$, then $H_{k-f}=H_{k}$. Consequently,

$$
\left\|H_{k}\right\|=\left\|H_{k-f}\right\| \leq\left\|M_{k-f}\right\|=\|k-f\|_{\infty},
$$

thus $\left\|H_{k}\right\| \leq \operatorname{dist}\left(k, H_{G}^{\infty}(\mathcal{L}(\mathcal{H}))\right)$. If $k \in L_{G}^{\infty}((\mathcal{H}))$, by Theorem 1.1 there exists $K \in L_{G}^{\infty}(\mathcal{L}(\mathcal{H}))$ such that $\|K\|_{\infty}=\left\|H_{k}\right\|$ and $\hat{K}_{h, k}(\gamma)=\int_{G} \overline{\gamma(x)}(k(x) h, k) d m(x)$, for every $\gamma \in(-P)$ and $h, k \in \mathcal{H}$. This implies that $k-K \in H_{G}^{\infty}(\mathcal{L}(\mathcal{H}))$, so $\operatorname{dist}\left(k, H_{G}^{\infty}(\mathcal{L}(\mathcal{H}))\right)=\left\|H_{k}\right\|$.

Let $a \in P$ and consider the subspace

$$
H_{a}^{2}(\mathcal{H})=\left\{f \in H_{G}^{2}(\mathcal{H}): f(x)=\sum_{0 \leq \gamma \leq a} \gamma(x) h_{\gamma}\right\}
$$

of $H_{G}^{2}(\mathcal{H})$. Let $P_{a}$ denote the orthogonal projection of $H_{G}^{2}(\mathcal{H})$ onto $H_{a}^{2}(\mathcal{H})$. If $k \in H_{G}^{2}(\mathcal{L}(\mathcal{H})), k(x)=\sum_{0 \leq \gamma \leq a} \gamma(x) k_{\gamma}$, define the (possibly unbounded) generalized Toeplitz operator $T_{a}=P_{a} M_{k} \mid H_{a}^{2}(\mathcal{H})$.

The following is our generalization of the Carathéodory-Fejér Theorem.

Corollary 3.2. Let $k \in H_{G}^{2}(\mathcal{L}(\mathcal{H})), k(x)=\sum_{0 \leq \gamma \leq a} \gamma(x) k_{\gamma}$ be such that $T_{a}$ is bounded. Then there exists $K \in H_{G}^{\infty}(\mathcal{L}(\mathcal{H}))$ such that $\|K\|_{\infty}=\left\|T_{a}\right\|$ and $\hat{K}(\gamma)=$ $k_{\gamma}$ for $0 \leq \gamma \leq a$.

Proof. Define $k_{a}(x)=\overline{a(x)} \sum_{0 \leq \gamma \leq a} \gamma(x) k_{\gamma}$. Then $\left\|H_{k_{a}}\right\|=\left\|T_{a}\right\|$ and one can apply Theorem 1.1 to $k_{a}$. Thus there exists $K_{a} \in L_{G}^{\infty}(\mathcal{L}(\mathcal{H}))$ such that $\left\|K_{a}\right\|_{\infty}=$ $\left\|H_{k_{a}}\right\|=\left\|T_{a}\right\|$ and $\hat{K}_{a}(\gamma)=k_{\gamma+a}$ for every $\gamma \in(-P)$. (We consider $k_{\gamma}=0$ for $\gamma \in(-P)$.) Define $K(x)=a(x) K_{a}(x)$. Then $K \in H_{G}^{\infty}(\mathcal{L}(\mathcal{H}))$, and $\hat{K}(\gamma)=k_{\gamma}$ for $0 \leq \gamma \leq a$.

\section{ACKNOWLEDGEMENT}

The author thanks Dan Timotin for helpful discussions during the preparation of this paper. 


\section{REFERENCES}

1. V.M. Adamjam, D.Z. Arov, and M.G. Krein, Infinite Hankel matrices and generalized Carathéodory-Fejér and Riesz problems, Functional Anal. Appl., Vol. 2(1968), 1-18. MR 38:2591

2. W.B. Arveson, Subalgebras of $C^{*}$-algebras, Acta Math., 123(1969), 141-224. MR 40:6274

3. W.B. Arveson, Interpolation problems in nest algebras, J. Funct. Anal., Vol. 3(1975), 208-233. MR 52:3979

4. M. Bakonyi, The extension of positive definite operator-valued functions defined on a symmetric interval of an ordered group, Proc. Amer. Math. Soc., Vol. 130, No. 5(2002), 1401-1406.

5. M. Bakonyi and D. Timotin, The intertwining lifting theorem for ordered groups, to appear in J. Functional Anal.

6. R. Bruzual and M. Dominguez, Extensions of operator valued positive definite functions and commutant lifting on ordered groups, J. Functional Anal., Vol. 185, No. 2(2001), 456-473. MR 2002g:43005

7. C. Carathéodory and L. Fejér, Über den Zusammenhang der Extremen von harmonischen Funktionen mit ihren Koeffizienten und Über den Picard-Landauschen Satz, Rend. Circ. Mat. Palermo, Vol. 32(1911), 193-217.

8. M. Dominguez, Interpolation and prediction problems for connected abelian groups, Integral Equations Operator Theory, Vol. 40, No. 2(2001), 212-230. MR 2002c:47030

9. C. Foias and A.E. Frazho, The Commutant Lifting Approach to Interpolation Problems, Birkhäuser Verlag, Basel-Boston-Berlin, 1990. MR 92k:47033

10. H. Helson and D. Lowdenslager, Prediction theory and Fourier series in several variables I, Acta Mathematica, Vol. 99(1958), 165-202. MR 20:4155

11. M.G. Krein, Sur le probléme de prolongement des functions hermitiniennes positives et continues, Dokl. Akad. Nauk. SSSR, Vol. 26(1940), 17-22. MR 2:361h

12. Z. Nehari, On bounded bilinear forms, Annals of Math., Vol. 65(1957), 153-162. MR 18:633f

13. V. Paulsen, Completely Bounded Maps and Dilations, Pitman Research Notes in Mathematics, Vol. 146, New York, 1986. MR 88h:46111

14. V.I. Paulsen, S.C. Power, and R.G. Smith, Schur products and matrix completions, J. Funct. Anal., Vol. 85(1989), 151-178. MR 90j:46051

15. L. Rodman, I. Spitkovsky, and H.J. Woerdeman, Carathéodory-Toeplitz and Nehari problems for matrix valued almost periodic functions, Trans. Amer. Math. Soc., Vol. 350, No. 6(1998), 2185-2227. MR 98h:47023

16. L. Rodman, I. Spitkovsky, and H.J. Woerdeman, Contractive extension problems for matrix valued almost periodic functions of several variables, J. Operator Theory, Vol. 47(2002), 3-35.

17. L. Rodman, I. Spitkovsky, and H.J. Woerdeman, Multiblock problems for almost periodic matrix functions of several variables, New York Journal of Math., Vol. 7(2001), 117-148. MR 2002h:42013

18. L. Rodman, I. Spitkovsky, and H.J. Woerdeman, Abstract band method via factorization, positive and band extensions of multivariable almost periodic matrix functions, and spectral estimation, to appear in the Memoirs of the AMS.

19. W. Rudin, Fourier Analysis on Groups, Interscience Publishers, New York, 1962. MR 27:2808

20. Z. Sasvári, Positive Definite and Definitizable Functions, Akademie Verlag, Berlin, 1994. MR 95c:43005

21. I. Spitkovsky and H.J. Woerdeman, The Carathéodory-Fejér problem for almost periodic functions, J. Funct. Anal., Vol. 115(1993), 281-293. MR 94f:47020

Department of Mathematics, Georgia State University, Atlanta, Georgia 30303-3083

E-mail address: mbakonyi@cs.gsu.edu 